\title{
Systematic Investigation of the Excited-State Properties of Anthracene-Dicarboxylic Acids
}

Jennifer M. Rowe, Jennifer M. Hay, William A. Maza, Robert C. Chapleski Jr., Erin Soderstrom, Diego Troya, and Amanda J. Morris*

Keywords: anthracene; photoluminescence; DFT calculations 


\section{ABSTRACT}

We report the photophysical properties of three anthracene-dicarboxylic acids - 1,4-anthracene dicarboxylic acid (1,4-ADCA), 2,6-anthracene dicarboxylic acid (2,6-ADCA) and 9,10anthracene dicarboxylic acid (9,10-ADCA) - in a series of polar aprotic solvents using steadystate absorption, steady-state emission spectroscopy and time-correlated single photon counting emission lifetime spectroscopy. The addition of carboxylic acid functional groups on the anthracene ring perturbs the electronic transitions oriented along the longitudinal and transverse axes to varying degrees, resulting in differences in the photophysical properties. The three anthracene derivatives exhibit very different excited-state properties in basic solution compared to acidic and neutral solutions. Density functional theory (DFT) calculations reveal that the lowest-energy ground-state structures of both 2,6-ADCA and 1,4-ADCA have dihedral angles between the carboxylic acids and aromatic planes of $\theta=0^{\circ}$. DFT calculations also indicate that there is a lower barrier for carboxyl group rotation for 1,4-ADCA (5.5 kcal/mol) than for 2,6-ADCA $(8 \mathrm{kcal} / \mathrm{mol})$ in the ground state. For $9,10-\mathrm{ADCA}$, the same dihedral angle increases to $\theta=56.6^{\circ}$. Time-dependent DFT calculations suggest that the carboxyl groups of 1,4-ADCA and 2,6-ADCA remain coplanar with the anthracene ring system in the excited state. In contrast, the calculations reveal significant changes between the ground and excited geometries for $9,10-\mathrm{ADCA}$ as the dihedrals decrease from $56.6^{\circ}$ to $27.7^{\circ}$ in the first excited state and puckering of the anthracene moiety of 9,10-ADCA is observed. 


\section{INTRODUCTION}

Organic photoactive molecules are of interest due to the tunability and environmental susceptibility of their excited-state properties, which affords a range of optoelectronic applications including organic light-emitting diodes (OLEDs), photovoltaic cells, organic field effect transistors, and fluorescent sensing elements. ${ }^{1-4}$ Anthracene is a polyaromatic hydrocarbon whose excited-state properties have been extensively studied. ${ }^{5}$ Due to its unique ground and excited-state properties, anthracene and its derivatives are often incorporated into devices such as OLEDs and organic semiconductor materials for numerous applications. ${ }^{6-9}$ However, in order to rationally tailor anthracene derivatives for a given application, an understanding of the effects of microenvironment and functionalization on the photophysical properties is essential.

The steady-state absorption and emission spectra of anthracene in THF are shown in Figure 1. The absorption spectrum of anthracene has two sets of bands in the $220 \mathrm{~nm}-280 \mathrm{~nm}$ and $290 \mathrm{~nm}-400 \mathrm{~nm}$ ranges, corresponding to three different $\pi \rightarrow \pi^{*}$ transitions. The absorption observed in each region displays vibronic structure and corresponds to one of two transition moments oriented along the molecular axes. The transition dipole of the high-energy transition centered around $256 \mathrm{~nm}$, corresponding to a ${ }^{1} \mathrm{~A} \rightarrow{ }^{1} \mathrm{~B}_{\mathrm{b}}$ transition (Platt notation), ${ }^{10}$ is polarized along the longitudinal axis. The low-energy transition centered around $386 \mathrm{~nm}$, corresponding to a ${ }^{1} \mathrm{~A} \rightarrow{ }^{1} \mathrm{~L}_{\mathrm{a}}$ transition is polarized along the transverse short axis, as illustrated in Figure 1.5,11,12 Another longitudinally polarized transition of ${ }^{1} \mathrm{~A} \rightarrow{ }^{1} \mathrm{~L}_{\mathrm{b}}$ character is also present, however, the oscillator strength of this transition is weak and is obscured by the much 
more intense ${ }^{1} \mathrm{~A} \rightarrow{ }^{1} \mathrm{~L}_{\mathrm{a}}$ transition band. The mirror-image relationship observed between the ${ }^{1} \mathrm{~A} \rightarrow{ }^{1} \mathrm{~L}_{\mathrm{a}}$ absorption bands and the ${ }^{1} \mathrm{~L}_{\mathrm{a}} \rightarrow{ }^{1} \mathrm{~A}$ emission band is indicative of a negligible change in the nuclear coordinates between the ${ }^{1} \mathrm{~A}$ ground state and ${ }^{1} \mathrm{La}_{\mathrm{a}}$ excited state from which the emission originates. ${ }^{11,13}$ Thus, according to the Frank-Condon principle, the geometry of the excited state of anthracene is similar to that of the ground state.

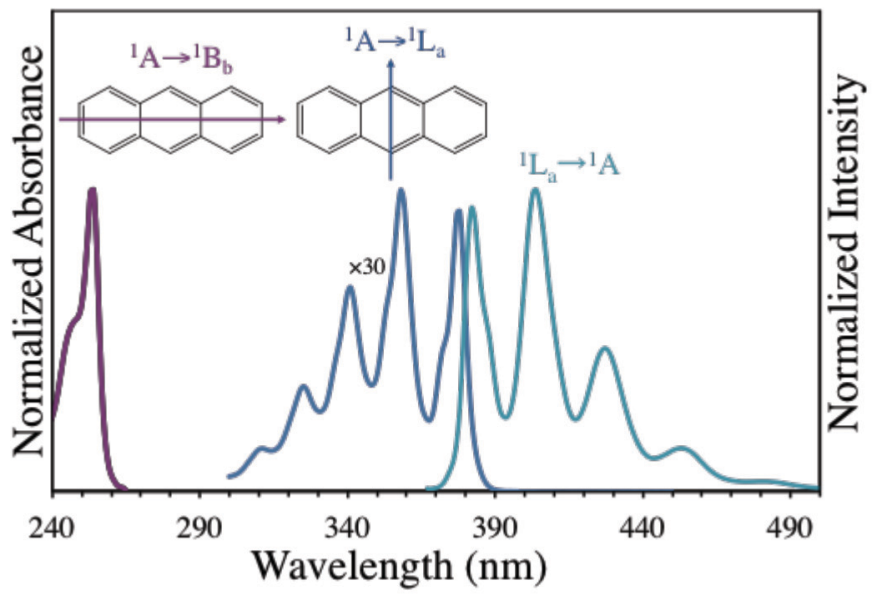

Figure 1. Absorbance spectrum of anthracene in THF showing the $\pi \rightarrow \pi^{*}$ transitions with dipole moments oriented along the longitudinal axis $\left({ }^{1} \mathrm{~A} \rightarrow{ }^{1} \mathrm{~B} b\right.$, purple) and across the short axis $\left({ }^{1} \mathrm{~A} \rightarrow{ }^{1} \mathrm{~L}_{\mathrm{a}}\right.$, dark blue) of anthracene and emission spectrum arising from the ${ }^{1} \mathrm{~L}_{\mathrm{a}} \rightarrow{ }^{1} \mathrm{~A}$ transition (light blue).

The positions of both the steady-state absorption and emission maxima of anthracene are independent of solvent polarity or hydrogen bonding at room temperature. ${ }^{14}$ Likewise, the fluorescence quantum yield $\left(\Phi_{\mathrm{fl}}\right)$ and fluorescence lifetime $\left(\tau_{\mathrm{f}}\right)$ of anthracene are insensitive to changes in solvation environment. ${ }^{15,16}$ For example, the fluorescence lifetimes measured in benzene, cyclohexane and ethanol are $4.29 \mathrm{~ns}, 5.24 \mathrm{~ns}$, and $5.1 \mathrm{~ns}$, respectively and the quantum yield of fluorescence is between 0.27 and 0.36 in the same solvents. ${ }^{17-20}$ 
The excited-state behavior of a carboxylic acid functionalized anthracene, 9anthracenecarboxylic acid (9-ACA) has been the topic of a number of reports. ${ }^{21-27}$ Changes in the emission spectra of 9-ACA have been argued to arise from acid-base equilibrium, solvent and concentration dependent dimerization and formation of higher order aggregates, as well as structural reorganization of the carboxylic acid relative to the anthracene ring in the excited state. ${ }^{22,24,26}$ In order to contribute to this discussion, we prepared a series of anthracene derivatives functionalized symmetrically with carboxylic acids; namely 2,6anthracenedicarboxylic acid (2,6-ADCA), 1,4-anthracenedicarboxylic acid (1,4-ADCA), and 9,10-anthracenedicarboxylic acid (9,10-ADCA). We characterize the effect of functionalization on the energetics of the ground and excited states of anthracene using steadystate absorption and emission spectroscopies, emission lifetime measurements, density functional theory (DFT) and time-dependent DFT (TDDFT) calculations. The results are interpreted in light of structural/conformational differences between the ground and excited states of each anthracene derivative.

\section{MATERIALS AND METHODS}

Materials: 2,6-anthracenedicarboxylic acid (2,6-ADCA), 1,4-anthracenedicarboxylic acid (1,4-ADCA), and 9,10-anthracenedicarboxylic acid (9,10-ADCA) were synthesized following previously reported procedures with minimal modifications and characterized by $1 \mathrm{H}$ NMR spectroscopy (Figures S12-S14). ${ }^{28-32}$ All other chemicals and solvents including anthracene (>99\%), $\mathrm{KOH}(85 \%), \mathrm{NH}_{4} \mathrm{OH}(25-30 \%)$, acetic 
acid (reagent grade > 99\%), dimethylformamide (HPLC grade > 99\%), dimethylacetamide (spectrophotometric grade $>99 \%$ ), tetrahydrofuran (ACS grade $>$ $99 \%$ ), ethyl acetate (HPLC grade $>99.9 \%$ ), acetone (HPLC grade $>99.5 \%$ ), and butanone (ACS grade $>99 \%$ ) were used as received without further purification from Alfa Aesar, Fisher Scientific, or Sigma-Aldrich.

Steady-state absorption spectroscopy: The steady-state absorption spectra of the ADCA derivatives were obtained using an Agilent Technologies 8453 UV-Vis diode array spectrophotometer ( $1 \mathrm{~nm}$ resolution) where the spectra were recorded with samples prepared in a $1 \mathrm{~cm}$ quartz cuvette. To determine the extinction coefficients of each compound, three solutions of known concentration were prepared separately, and then each was diluted three times. The UV-vis absorbance of each solution was measured and the absorbance at a fixed wavelength was plotted vs. concentration. The extinction coefficient was determined by averaging the slopes of the lines-of-best-fit obtained from each of the three plots.

\section{Steady-state emission spectroscopy and time-resolved emission lifetimes: All}

samples were prepared at concentrations below $10 \mu \mathrm{M}$ to reduce aggregation effects and purged with argon before measurements were performed. Time-resolved fluorescence lifetimes were obtained via the time-correlated single photon counting technique (TCSPC) with a modified QuantaMaster Model QM-200-4E emission spectrophotometer from Photon Technology, Inc. (PTI) equipped with a $350 \mathrm{~nm}$ LED and a Becker \& Hickl GmbH PMH-100 PMT detector with time resolution of $<220$ ps full width at half maximum (FWHM). ${ }^{33}$ Florescence lifetime decays were deconvoluted from the time-dependent florescence signal 
and the instrument response function using the fluorescence decay analysis software, DecayFit, available online (Fluortools, www.fluortools.com, Figures S9-S11).

Quantum yields of fluorescence and steady-state emission spectra of the ADCA compounds were measured in ethyl acetate, tetrahydrofuran (THF), butanone, acetone, dimethylacetamide (DMA), dimethylformamide (DMF), acidic (acetic acid) DMF and basic (NaOH) DMF. The steady-state emission spectra were obtained using the same QuantaMaster Model QM-200-4E where the sample compartment was replaced with an integrating sphere (PTI). The excitation light source was a $75 \mathrm{~W}$ Xe arc lamp (Newport). The detector was a thermoelectrically cooled Hamamatsu 1527 photomultiplier tube (PMT). All measurements were performed in triplicate using three separately prepared solutions of ADCA in each solvent with absorbance values of $0.08-0.09$ at the excitation wavelength.

Theoretical calculations: Radiative decay rates were calculated by applying the Strickler-Berg method using the PhotochemCAD 2.1 software. All electronic structure calculations were carried out with the Gaussian 09 suite of programs. ${ }^{43}$ The DFT calculations presented in this work were carried out at the B3LYP/6-31G* level. Sample calculations using the M06 density functional, and the larger $6-311 \mathrm{G}^{*}$ basis set were used to corroborate key results at the B3LYP/ 6-31G* level. Ground-state potential-energy contours correspond to relaxed scans in which the relevant dihedral angles between the carboxylic acid groups and the anthracene moiety are allowed to vary at $15^{\circ}$ steps while the rest of the coordinates are optimized. Potential-energy contours for the carboxylic acids in the first excited state were obtained using time-dependent B3LYP/6-31G* calculations, directly using the geometries obtained from ground-state dihedral scans. 
Determination of acid association constants: Ground-state acid association constants $(K)$ of the ADCA derivatives were determined via absorption spectroscopy by monitoring the change in absorption (corrected for dilution) as a function of added acid concentration. Samples of the three acids were prepared in solutions of water with 0,10 and $30 \mathrm{~mol} \%$ DMF and were titrated using $\mathrm{NaOH}$ and $\mathrm{HCl}$. The absorption value near the isosbestic point at each addition of acid was plotted as a function of acid concentration and the data was fit to Equation 1,

where $A_{i}$ is the absorbance at a given concentration of acetic acid, $A_{\max }$ and $A_{\min }$ are the absorbance values for the fully protonated and deprotonated species, respectively, $n$ is the number of protons associated with a given acid association constant, $K . A_{\mathrm{i}}$ values were fit to Equation 1 with $K$ as a variable parameter. Fixing $n$ to 2 yielded the most reasonable fit of the data. The $\mathrm{p} K$ values were plotted versus mol\% DMF and the $\mathrm{p} K$ value of each compound in pure DMF was determined by extrapolating to $100 \mathrm{~mol} \%$ DMF (Figures S6-S8). Excited-state acid association constants $\left(\mathrm{p} K^{*}\right)$ were predicted using the Förster cycle, (Equation 2),

where and correspond to the wavenumber of the $0-0$ transitions of $\mathrm{A}^{-}$and $\mathrm{AH}$, respectively. The wavenumber corresponding to the $0-0$ transition was estimated by averaging the wavenumbers at the absorption and emission maxima (Equation 3). ${ }^{4}$

\section{RESULTS}


The steady-state absorption and emission spectra of 2,6-ADCA (a,b), 1,4-ADCA (c,d) and 9,10-ADCA (e,f) measured in THF are shown in Figure 2. 2,6-ADCA displays vibronically structured absorption and emission bands. Although the structure of the emission spectrum closely resembles that of anthracene, the absorption spectrum differs from anthracene in both the number of lower-energy $(\sim 320 \mathrm{~nm}-420 \mathrm{~nm}$ for 2,6-ADCA and $285 \mathrm{~nm}-385 \mathrm{~nm}$ for anthracene) absorption bands and their intensities. The absorption spectrum of 1,4-ADCA appears broad with subtle structural features at $360 \mathrm{~nm}$ and $370 \mathrm{~nm}$ and the emission spectrum is broad and structureless. 9,10-ADCA exhibits vibronically structured absorption band, similar in shape to anthracene, while the emission spectrum is broad and diffuse. Both the absorption and emission spectra of each derivative are bathochromically shifted relative to anthracene (Table 1). Additionally, the ${ }^{1} \mathrm{~A} \rightarrow{ }^{1} \mathrm{~B}_{\mathrm{b}}$ absorption peak of $1,4-\mathrm{ADCA}$ at $240 \mathrm{~nm}$ is significantly broadened and relative to anthracene and is split into two peaks. 2,6-ADCA exhibits a Stokes shift $\left(584 \mathrm{~cm}^{-1}\right.$ in THF) that is slightly larger than that of anthracene (208 $\mathrm{cm}^{-1}$ in THF). 1,4-ADCA and 9,10-ADCA display large Stokes shifts $\left(4,803 \mathrm{~cm}^{-1}\right.$ and 3,667 $\mathrm{cm}^{-1}$ in THF) compared to anthracene. The observed lifetimes of each isomer in THF are considerably longer than the $4.9 \pm 0.1 \mathrm{~ns}$ fluorescence lifetime of anthracene in the same solvent (Table 2). 

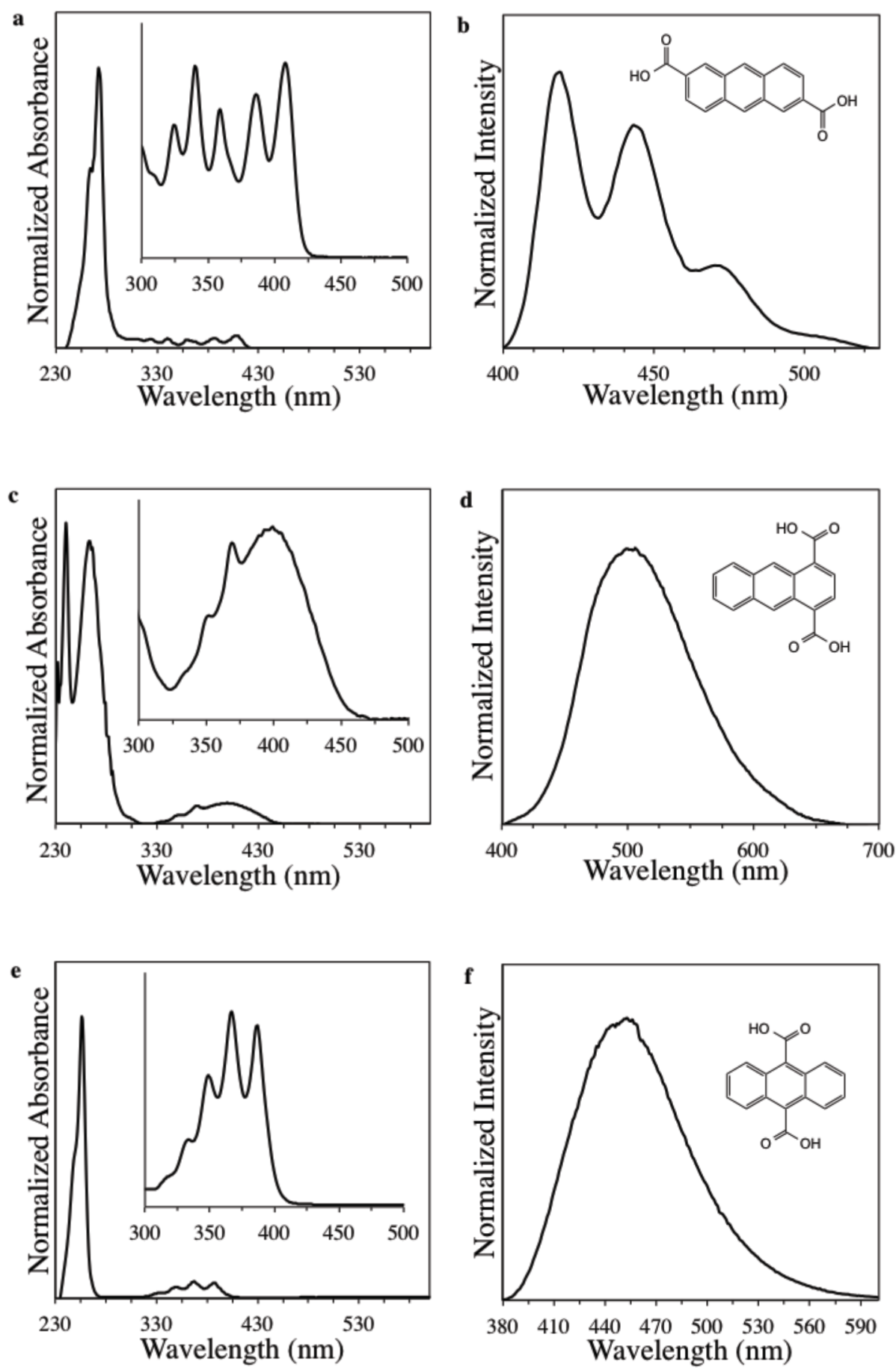

Figure 2. Absorption and emission spectra of 2,6-ADCA (a,b) 1,4-ADCA (c,d) and 9,10$\operatorname{ADCA}(\mathrm{e}, \mathrm{f})$ in THF. 
In order to probe the effects of solvent polarity on the photophysical properties of the anthracene derivatives, the absorption and emission spectra as well as the lifetimes and quantum yields were measured in a series of polar aprotic solvents. Neither the absorption nor emission spectra of 9,10-ADCA are affected by solvent polarity. The absorption spectrum of 9,10-ADCA displays anthracene-like vibrational structure in all neat solvents tested. The emission spectrum is broad and diffuse with a around $455 \mathrm{~nm}$ in neat solvents, in which is bathochromically shifted by $14 \mathrm{~nm}$ (Figure S3, Table S1). The observed Stokes shift is 3,796 $\mathrm{cm}^{-1} \pm 114$ in THF, ethyl acetate, butanone, acetone and DMA (Table S1). Similarly, solvent polarity has no significant effect on the absorption or emission spectra 2,6-ADCA, as the maxima do not shift significantly between $\operatorname{THF}(\varepsilon=7,=409 \mathrm{~nm},=419 \mathrm{~nm})$, and DMA $(\varepsilon=$ $38,=408 \mathrm{~nm},=421 \mathrm{~nm}$, Figure S1, Table S1). In contrast to the other two isomers, the absorption band of 1,4-ADCA is hypsochromically shifted by $10 \mathrm{~nm}$, from $396 \mathrm{~nm}$ in THF ( $\varepsilon$ $=7)$ to $386 \mathrm{~nm}$ for DMA ( $\varepsilon=38$, Figure S2, Table S1), and the emission maximum is bathochromically shifted by $8 \mathrm{~nm}$ from $489 \mathrm{~nm}$ in THF to $497 \mathrm{~nm}$ in DMA. The absorption and emission spectra of 1,4-ADCA remain broad and diffuse in all of the neat solvents explored.

The fluorescence quantum yields $\left(\Phi_{\mathrm{fl}}\right)$ and fluorescence lifetimes $\left(\tau_{\mathrm{f}}\right)$ along with the experimental radiative $\left(\mathrm{k}_{\mathrm{r}, \mathrm{exp}}\right)$ and non-radiative $\left(\mathrm{k}_{\mathrm{nr}}\right)$ rate constants and calculated $\left(\mathrm{k}_{\mathrm{r}, \mathrm{calc}}\right)$ rate constant obtained from the Strickler-Berg equation for the three ADCA derivatives in the various solvents are listed in Table 2 and Table S2. No trends are observed between solvent polarity and fluorescence quantum yields over the solvent series (Table S2). In general, the 
fluorescence lifetimes are largely independent of solvent polarity but are considerably shorter in basic conditions. ${ }^{17-20}$
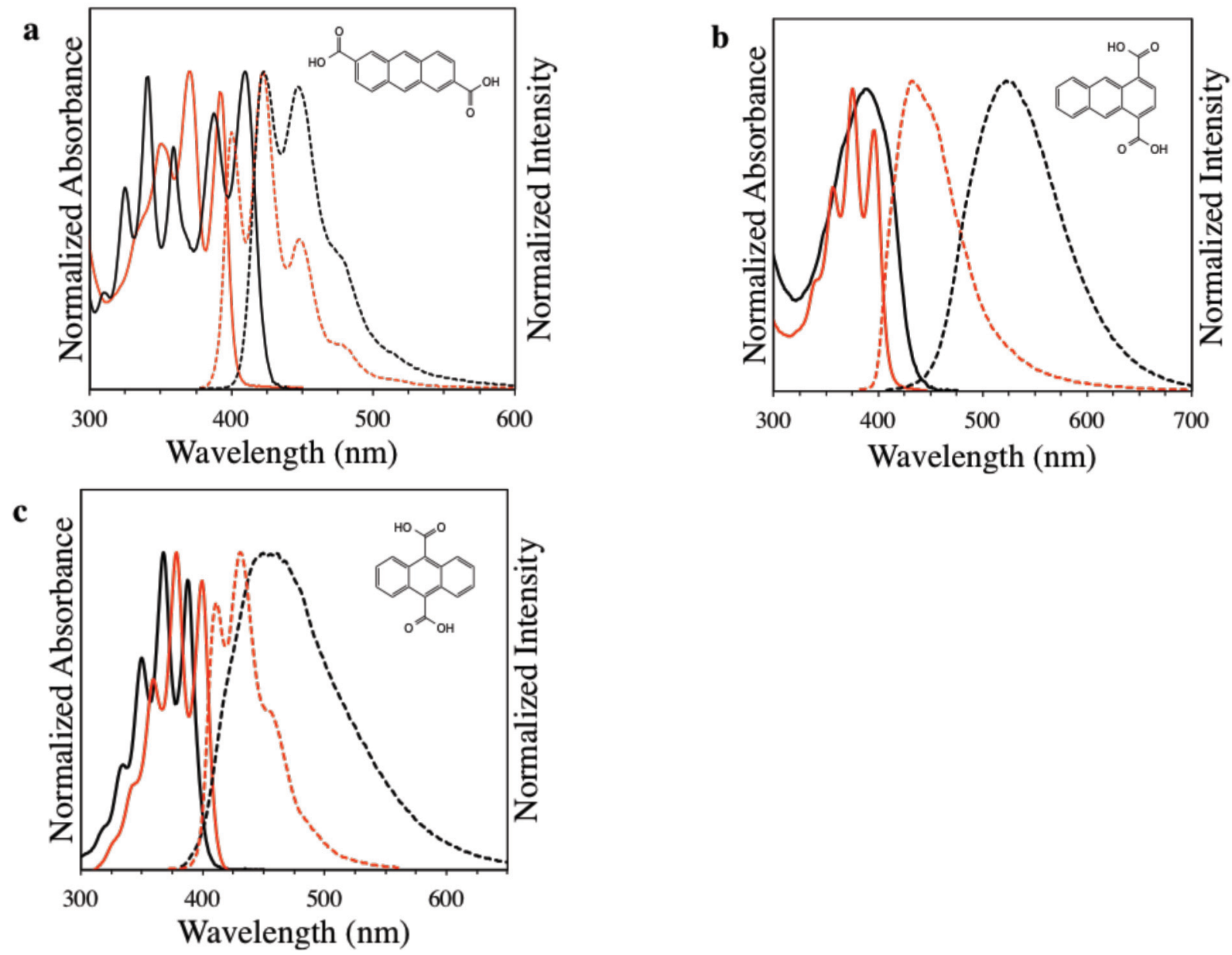

Figure 3. Absorption and emission spectra of 2,6-ADCA (a), 1,4-ADCA (b) and 9,10-ADCA (c) acidic DMF (black) and basic DMF (red).

To further explore how the protonation state of the carboxylic acid substituents affects the excited-state properties of the anthracene derivatives, photophysical measurements were carried out in acidic DMF and basic DMF (Figure 3, Table 2). All three isomers exhibit anthracene-like vibronic structure in the absorption spectra recorded in basic DMF. The emission spectrum of 2,6-ADCA displays more defined vibronic structure in basic solution 
compared to acidic. The emission spectrum of 1,4-ADCA remains broad and structureless in both basic and acidic environments. On the other hand, 9,10-ADCA exhibits a broad, diffuse emission spectrum in acidic DMF, but in basic DMF anthracenic vibronic structure is observed. The absorption spectra of 1,4-ADCA and 9,10-ADCA are hypsochromically shifted in basic DMF relative to acidic, while that of 2,6-ADCA is bathochromically shifted in basic solution. Additionally, the emission spectrum of each of the derivatives is hypsochromically shifted under basic conditions compared to acidic. The Stokes shifts decrease considerably with solvent $\mathrm{pH}$, going from $809 \mathrm{~cm}^{-1}$ to $510 \mathrm{~cm}^{-1}$ for 2,6-ADCA, $6,484 \mathrm{~cm}^{-1}$ to $1,997 \mathrm{~cm}^{-1}$ for 1,4-ADCA, and from $3,534 \mathrm{~cm}^{-1}$ to $610 \mathrm{~cm}^{-1}$ for 9,10-ADCA (Table 1). The quantum yields of 2,6-ADCA and 1,4-ADCA decrease considerably under basic conditions compared to acidic solution, while that of 9,10-ADCA decreases only by about half (Table 2).

Table 1. Summary of absorption and emission data for the ADCAs

\begin{tabular}{|c|c|c|c|c|c|}
\hline & Solvent & $(\mathbf{n m})$ & $(\mathrm{nm})$ & Stokes $\left(\mathrm{cm}^{-1}\right)$ & $\mathrm{E}_{0,0}(\mathrm{eV})$ \\
\hline \multirow[t]{3}{*}{ 2,6-ADCA } & THF & 409 & 419 & 584 & 3.0 \\
\hline & Acidic DMF & 409 & 423 & 809 & 3.0 \\
\hline & Basic DMF & 392 & 400 & 510 & 3.1 \\
\hline \multirow[t]{3}{*}{ 1,4-ADCA } & THF & 396 & 489 & 4803 & 2.8 \\
\hline & Acidic DMF & 390 & 522 & 6484 & 2.8 \\
\hline & Basic DMF & 396 & 430 & 1997 & 2.9 \\
\hline \multirow[t]{3}{*}{ 9,10-ADCA } & THF & 387 & 451 & 3667 & 3.1 \\
\hline & Acidc DMF & 389 & 451 & 3534 & 3.1 \\
\hline & Basic DMF & 400 & 410 & 610 & 3.1 \\
\hline
\end{tabular}


is the lowest energy maximum in the absorbance spectrum, and is the highest energy maximum in the fluorescence spectrum.

To quantify the degree of protonation in both the ground and excited states as a function of $\mathrm{pH}$, the ground-state acid association constants $(K)$ of the ADCAs were determined by absorption spectroscopy (Figures $\mathrm{S6-S8}$, equation 2) and $\mathrm{p} K$ values of 17.6, 17.9 and 19.4, for 2,6-ADCA, 1,4-ADCA and 9,10-ADCA, respectively, were obtained in the ground state. The excited-state acid association constants $\left(\mathrm{p} K^{*}\right)$ were then calculated in DMF from the Förster cycle (vide supra), and found to be 23.0, 23.2 and 23.8, accordingly. Thus, in neutral solutions, the ADCA derivatives are predominantly protonated in both the ground state and the excited state. 
Table 2. Summary of the lifetime and quantum yield data for ADCAs

\begin{tabular}{|c|c|c|c|c|c|c|c|c|}
\hline & Solvent & $\tau_{f}(\mathbf{n s})$ & $\tau_{0}(\mathrm{~ns})$ & $\Phi_{f l}$ & $\begin{array}{c}\mathbf{k} \\
\mathbf{r , e x p} \\
\left(10^{-7} \mathbf{S}^{-}\right. \\
1)\end{array}$ & $\begin{array}{c}\mathbf{k} \\
{ }_{\text {nr,exp }} \\
\left(10^{-7} \mathbf{S}^{-}\right. \\
1)\end{array}$ & $\begin{array}{c}\tau_{0, \text { calc }} \\
(\mathbf{n s})\end{array}$ & $\begin{array}{c}\mathbf{k} \\
\mathbf{r , c a l c} \\
\left(\mathbf{1 0}^{-7} \mathbf{s}^{-1}\right)\end{array}$ \\
\hline \multirow[t]{3}{*}{ 2,6-ADCA } & THF & $\begin{array}{c}12.3 \pm \\
0.7\end{array}$ & $\begin{array}{c}15.8 \pm \\
0.08\end{array}$ & $0.78 \pm 0.074$ & 0.063 & 0.018 & - & - \\
\hline & $\begin{array}{l}\text { Acidic } \\
\text { DMF }\end{array}$ & $\begin{array}{c}12.5 \pm \\
0.4\end{array}$ & $14.6 \pm 0.1$ & $0.82 \pm 0.080$ & 0.068 & 0.015 & 10.7 & 0.093 \\
\hline & Basic DMF & $\begin{array}{c}2.9 \pm \\
0.02\end{array}$ & $\begin{array}{c}26.4 \pm \\
0.03\end{array}$ & $0.11 \pm 0.018$ & 0.038 & 0.307 & 7.6 & 0.132 \\
\hline \multirow[t]{3}{*}{ 1,4-ADCA } & THF & $\begin{array}{c}19.2 \pm \\
1.0\end{array}$ & $19.2 \pm 1.0$ & $0.61 \pm 0.068$ & 0.032 & 0.020 & 23.9 & 0.042 \\
\hline & $\begin{array}{l}\text { Acidic } \\
\text { DMF }\end{array}$ & $\begin{array}{c}12.5 \pm \\
0.1\end{array}$ & $12.5 \pm 0.1$ & $0.19 \pm 0.042$ & 0.031 & 0.049 & 21.2 & 0.047 \\
\hline & Basic DMF & $1.0 \pm 0.1$ & $1.0 \pm 0.1$ & $\begin{array}{c}0.066 \pm \\
0.015\end{array}$ & 0.066 & 0.930 & 15.6 & 0.064 \\
\hline \multirow{3}{*}{$\begin{array}{l}9,10- \\
\text { ADCA }\end{array}$} & THF & $8.8 \pm 0.1$ & $13.3 \pm 0.2$ & $0.66 \pm .085$ & 0.075 & 0.039 & 21.0 & 0.048 \\
\hline & $\begin{array}{l}\text { Acide } \\
\text { DMF }\end{array}$ & $5.7 \pm 0.3$ & $14.3 \pm 0.1$ & $0.40 \pm 0.05$ & 0.070 & 0.110 & 16.3 & 0.061 \\
\hline & Basic DMF & $\begin{array}{c}2.2 \pm \\
0.04\end{array}$ & $11.6 \pm 0.4$ & $0.19 \pm 0.015$ & 0.086 & 0.370 & 12.6 & 0.079 \\
\hline $\begin{array}{c}\text { Anthracen } \\
\mathrm{e}\end{array}$ & THF & $4.9 \pm 0.1$ & $\begin{array}{c}12.3 \pm \\
0.05\end{array}$ & $0.40 \pm 0.01$ & 0082 & 0.120 & 12.6 & 0.079 \\
\hline
\end{tabular}

In addition to solvent polarity and protonation state, geometry can have a significant effect on photophysical properties. DFT and TDDFT calculations were performed to obtain the energies and geometries of the three compounds as a function of the dihedral angles (see Figure S4 for a definition of he dihedral angles) between the carboxylic acid groups and the 
anthracene ring system (Figure 4) both in the ground state and in the excited state. The potential energy contours for the 2,6-ADCA isomer in the ground state (Figure 4a) show that the lowest-energy conformation occurs when the carboxylic acids and the anthracene moiety are coplanar. In the excited state, the lowest-energy geometry does not change with respect to the ground state and the molecule remains planar (Figure 4b). Similar results are obtained for the 1,4-ADCA isomer, where coplanarity of the carboxylic acids and anthracene moieties is observed in both the ground (Figure 4c) and the first excited state (Figure 4d). On the other hand, the results for the 9,10-ADCA isomer are strikingly different (Figure 4e, and 4f). For this isomer, the lowest-energy conformation does not exhibit the carboxylic acids in the same plane as the aromatic rings in either the ground or the first excited state. Full geometry optimizations reveal that the lowest-energy structure for the ground state 9,10-isomer has dihedral angles of $56.6^{\circ}$, and these dihedrals decrease to $27.7^{\circ}$ in the first excited state (Figure 5). Furthermore, the anthracene moiety in the 9,10-isomers seems to undergo a noticeable puckering upon excitation that is completely absent in the other two isomers.

Close examination of the energy contours in Figure 4 reveals that, interestingly, there seems to be a lower barrier for carboxyl rotation for 1,4-ADCA than for 2,6-ADCA in the ground state. Indeed, when one of the carboxylic acid groups is coplanar to the anthracene moiety, the barrier for full rotation of the other carboxylic acid group is about $5.5 \mathrm{kcal} / \mathrm{mol}$ in 1,4-ADCA, but around $8 \mathrm{kcal} / \mathrm{mol}$ in 2,6-ADCA. The most energetically favorable conformations of both 2,6-ADCA and 1,4-ADCA have dihedral angles of $\theta=0^{\circ}$ and both have a maximum energy when $\theta=90^{\circ}$. 

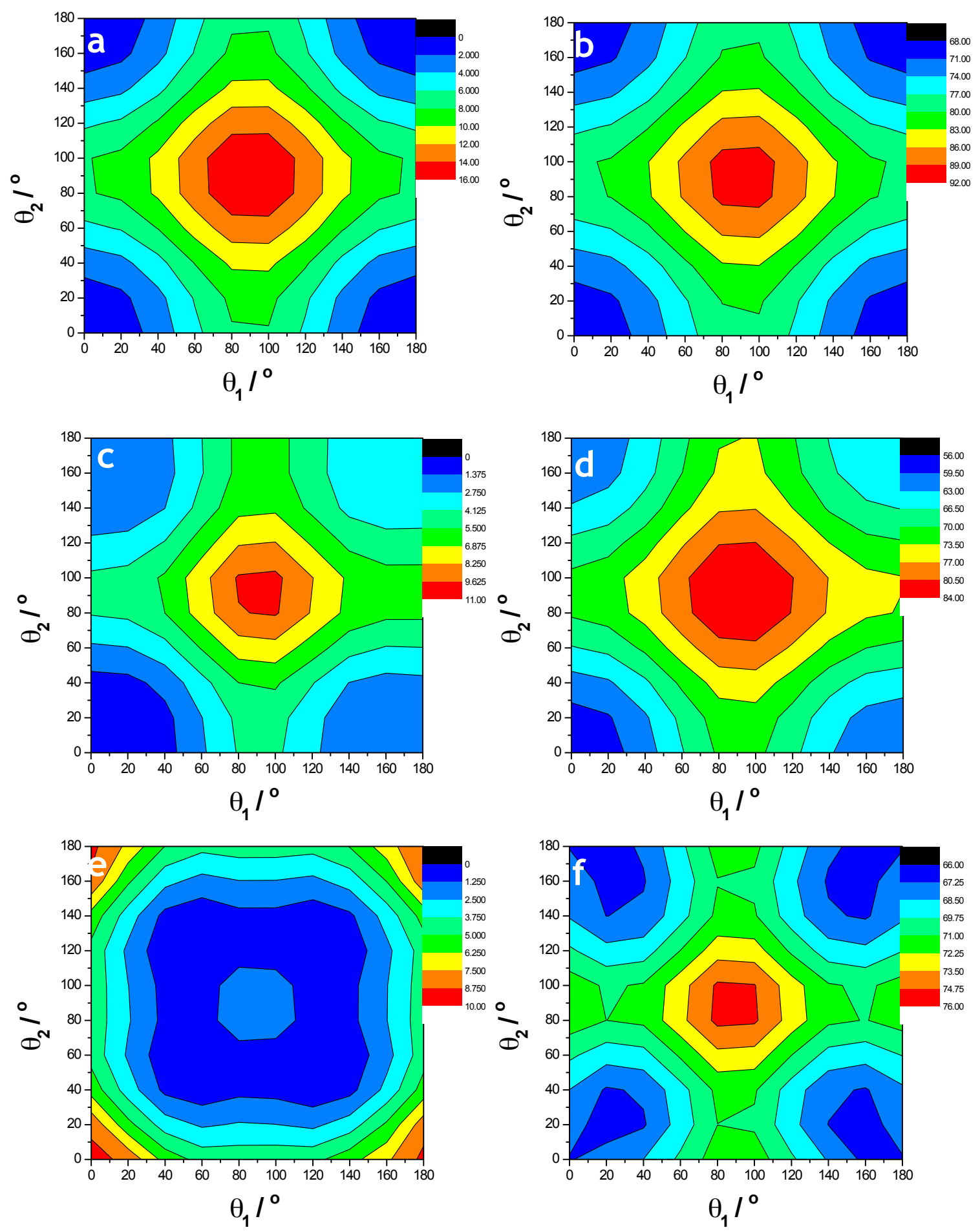

Figure 4. DFT calculated energies as a function of dihedral angles between $-\mathrm{COOH}$ groups and anthracene. Ground state of 2,6-ADCA (a), 1,4-ADCA (c), and 9,10-ADCA (e), and first 
excited state of 2,6-ADCA (b), 1,4-ADCA (d), and 9,10-ADCA (f). Energy scales for each plot are shown in $\mathrm{kcal} / \mathrm{mol}$ relative to the minimum energy of the ground state. (See Figure S4 for a definition of the dihedral angles.)

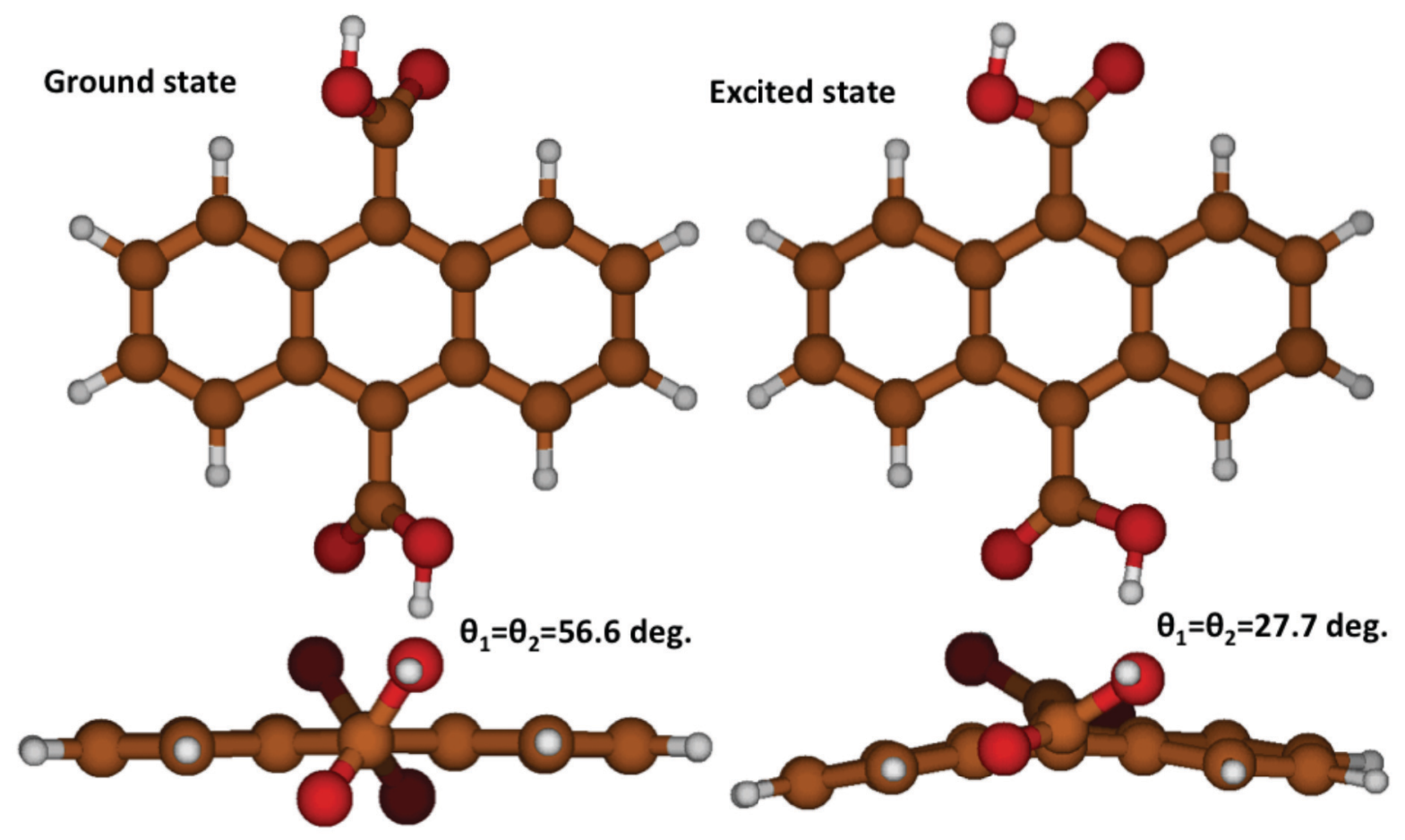

Figure 5. DFT calculated lowest-energy structures of the 9,10-ADCA molecule in the ground state (left) and the first excited state (right).

\section{DISCUSSION}

As expected, the photophysical characteristics of anthracene are sensitive to the position of the carboxylic acid functionalities on the ring system. Additionally, the protonation state of the acid groups further alters the excited-state properties of each anthracene derivative. The question then becomes, what is the main process that governs the photophysics of each isomer; 
acid-base equilibrium, solvent, formation of higher order aggregates, or excited-state structural reorganization? Experimentally determined rates of radiative decay can be compared with rates calculated by the Strickler-Berg equation to give insight into the intramolecular and intermolecular interactions that may impact the photophysical properties of the fluorophore. The Strickler-Berg equation (Equation 4) $)^{5}$ extends Einstein's formalism for transition probabilities of atomic absorption and emission to polyatomic molecules. From this equation, the intrinsic lifetime of a molecule, i.e. the radiative lifetime in the absence of intermolecular forces, can be estimated. The Stickler-Berg relation has accurately predicted the lifetimes of a number of molecules; however, it makes a few assumptions that limit its application in certain cases.

The relationship is based on the Born-Oppenheimer approximation, which assumes that the electronic transition dipole-moment operator does not depend on nuclear coordinates. Furthermore, it assumes that emission occurs only from the lowest-energy excited state. The predictive power of the Strickler-Berg relationship is, therefore, limited by large changes in the nuclear configuration upon generating the excited state, as well as intermolecular interactions between the chromophore and its environment. ${ }^{34}$ These latter assumptions are the origin of the discrepancy in this work between the calculated $\mathrm{k}_{\mathrm{r}, \text { calc }}$ obtained from the Strickler-Berg and the experimental $\mathrm{k}_{\mathrm{r}, \text { exp }}$ obtained from the measured fluorescence lifetime and quantum yields of 1,4-ADCA and 9,10-ADCA, while other phenomena are likely responsible for the discrepancies for 2,6-ADCA (Table 2, Table S2). 
Neither solvent polarity nor hydrogen bonding character alters the photophysics of 2,6ADCA. Thus, it is unlikely that intermolecular interactions play a significant role in altering the excited-state properties of the anthracene moiety of this isomer. The absorption spectra of 2,6-ADCA recorded in acidic and neat solvents display distinct vibronic structure, different from that observed in basic solution, which closely resembles the structure of the unsubstituted anthracene molecule. Similar observations have been reported in the absorption spectrum of 2napthalene carboxylic acid (2-NCA) and 2-anthracenecarboxylic acid (2-ACA). ${ }^{35}$ In general, electronic transitions polarized along a given axis are more strongly affected by derivatization of the molecule in the same direction. For example, the dissimilarity of the vibronic bands in the absorption spectrum of 2-ACA compared with anthracene was attributed to stabilization of the ${ }^{1} \mathrm{~A} \rightarrow{ }^{1} \mathrm{~L}_{\mathrm{b}}$ longitudinally polarized low-energy transition. The ${ }^{1} \mathrm{~A} \rightarrow{ }^{1} \mathrm{~L}_{b}$ transition is weak in unsubstituted anthracene and the corresponding absorption bands are obscured by the more intense ${ }^{1} \mathrm{~A} \rightarrow{ }^{1} \mathrm{~L}_{\mathrm{a}}$ absorption bands. Addition of a second carboxylic acid group, which further extends the length of the molecule, would likely result in greater stabilization of the ${ }^{1} \mathrm{~A} \rightarrow{ }^{1} \mathrm{Lb}_{b}$ transition. Accordingly, the bands assigned to ${ }^{1} \mathrm{~A} \rightarrow{ }^{1} \mathrm{~L}_{b}$ appear with greater intensity in the absorbance spectrum of 2,6-ADCA. DFT calculations of the lowest energy ground-state configuration of 2,6-ADCA suggest a coplanar arrangement between the carboxylic acids and the anthracene macrocycle (Figure 4a). This is in agreement with that observed in crystal structures of 2-ACA. ${ }^{36}$ The coplanar arrangement allows for resonance interactions between the acid groups and the aromatic ring system to occur. These resonance interactions increase the dipole moment, and thus, the oscillator strength of the ${ }^{1} \mathrm{~A} \rightarrow{ }^{1} \mathrm{~L}_{b}$ transition, resulting in the appearance of the ${ }^{1} \mathrm{~A} \rightarrow{ }^{1} \mathrm{~L}_{b}$ bands. ${ }^{35}$ The appearance of these strong ${ }^{1} \mathrm{~A} \rightarrow{ }^{1} \mathrm{~L}_{b}$ transition bands 
may contribute to the inconsistency between the $\mathrm{k}_{\mathrm{r} \text {,calc }}$ values and the $\mathrm{k}_{\mathrm{r}, \exp }$ values, since the integral of the area under the non-emissive ${ }^{1} \mathrm{~A} \rightarrow{ }^{1} \mathrm{~L}_{\mathrm{b}}$ absorption bands is a component of the Strickler-Berg equation.

The emission spectrum of 2,6-ADCA in neutral and basic solutions is not a mirror image of the absorbance spectrum. Although it displays anthracenic vibronic structure in all solvent conditions, the number of vibronic transitions in the emission is reduced compared to the absorption spectrum. This is not entirely unexpected, as ${ }^{1} \mathrm{~L}_{\mathrm{a}}$ is the only emissive state, and the additional vibronic structure is assumed to be due to absorption into the ${ }^{1} \mathrm{~L}_{\mathrm{b}}$ state..$^{35}$ The vibronic structure is indicative of little intermolecular reorganization in the excited state. The slight bathochromic shift in the absorption spectrum relative to anthracene is attributed to inductive effects imposed by the presence of the electron withdrawing functional groups on the polyaromatic ring. -

At higher $\mathrm{pH}$ values, the carboxylate groups of 2,6-ADCA are less likely to participate in resonance interactions, leading to further decoupling from the aromatic ring system and giving rise to anthracenic vibronic structure in the absorption spectrum. ${ }^{37}$ Indeed, the vibronic structure in the absorbance spectrum of deprotonated 2,6-ADCA ${ }^{2-}$ more closely resembles that of anthracene, but is bathochromically shifted $\sim 19 \mathrm{~nm}$ relative to anthracene. Thus, the carboxylic acid groups affect the aromatic system through inductive effects but do not participate in resonance interactions when deprotonated.

Contrary to 2,6-ADCA, both the absorption and emission spectra of 1,4-ADCA in neutral solvents lack vibronic structure and are diffuse. ${ }^{35}$ Similar observations have been 
reported for 1-anthracenecarboxylic acid (1-ACA). ${ }^{35}$ DFT calculations predict a lowest energy ground state geometry in which the carboxylic acid groups are coplanar with anthracene (Figure 4c). This conformation increases resonance interactions between the overlapping orbitals of the $\pi$-system and carboxyl groups, introducing charge-transfer (CT) character into the ${ }^{1} \mathrm{~A} \rightarrow{ }^{1} \mathrm{~L}_{\mathrm{a}}$ transition (polarized along the short axis) and electron density shifts toward the functionalized ring. The resulting shift of electron density results in loss of vibronic structure in both the absorption and emission spectra. The magnitude of the Stokes shift of 1,4-ADCA increases with the polarity of the solvent, i.e. THF $<\mathrm{DMF} \approx \mathrm{DMA}$. Closer inspection of the spectroscopic data reveals that, as solvent polarity increases, an overall bathochromic shift of $\sim 19 \mathrm{~nm}$ is observed in the emission spectrum. Because the carboxyl groups of 1,4-ADCA introduce $\mathrm{CT}$ character into the ${ }^{1} \mathrm{~A} \rightarrow{ }^{1} \mathrm{~L}_{\mathrm{a}}$ transition, the absorption spectrum is sensitive to solvent interactions with the functional groups. As solvent polarity increases, the excited state is stabilized due to solvent-solute induced dipolar interactions, giving rise to the bathochromic shift observed in the emission spectrum. ${ }^{35,38}$ Therefore, solvent relaxation about the weaker excited-state dipole likely contributes to the large Stokes shifts. The coplanar arrangement of the carboxylic acids in the ground state, along with the lack of vibronic structure in the emission spectrum, suggests that the resonance interactions between the carboxylic acids and aromatic ring system in the ground state are conserved in the excited state, which is corroborated by the coplanar lowest-energy structure found in the first excited state (Figure $4 d)$.

In basic solution, the absorbance spectrum of $1,4-\mathrm{ADCA}^{2-}$ is vibronically structured, while the emission spectrum is still broad and diffuse. Similar observations were reported for 
1-ACA- ${ }^{-35}$ In both molecules, the deprotonated carboxyl groups do not contribute to resonance forms of the anion in the ground state and consequently, the absorbance spectra are vibronically structured. There are several factors that may contribute to the loss of structure in the emission spectrum. For $1-\mathrm{ACA}^{-}$, the authors proposed that a shift in electron density upon promotion to the ${ }^{1} \mathrm{~L}_{\mathrm{a}}$ excited state in the direction of the carboxylate group introduces some $\mathrm{CT}$ character between the functional group and anthracene moiety, resulting in broadening of the emission spectrum. ${ }^{35}$ It is plausible that such phenomena also contribute to the loss of structure observed in the emission spectrum of 1,4-ADCA ${ }^{2-}$. The Stokes shift of 1,4-ADCA ${ }^{2-}\left(1997 \mathrm{~cm}^{-}\right.$ $\left.{ }^{1}\right)$ is large in comparison with the deprotonated $2,6-\mathrm{ADCA}^{2-}\left(510 \mathrm{~cm}^{-1}\right)$ and $9,10-\mathrm{ADCA}^{2-}$ $\left(610 \mathrm{~cm}^{-1}\right)$. A relatively large change in molecular dipole moment upon excitation would result in such a substantial Stokes shift. Solvent relaxation may also have a significant effect on the fluorescence spectrum. Because the energy of the relaxed excited state is lower than that of the initial Frank-Condon excited state, thermodynamic equilibration with the surrounding solvent would contribute to the loss of structure and bathochromic shift in the emission spectrum of 1,4-ADCA ${ }^{2-}$. Lastly, it may be possible that the carboxylate groups can easily rotate, which would also give rise to a large Stokes shift (relative to both 2,6- $\mathrm{ADCA}^{2-}$ and 9,10-ADCA ${ }^{2-}$ ) and broad emission spectrum due to nuclear reorganization. Unfortunately, the excited state geometry of $1,4-\mathrm{ADCA}^{2-}$ could not be predicted computationally, as energy calculations for the deprotonated molecule encountered severe convergence problems.

In neat solvents, the absorption spectrum of 9,10-ADCA displays anthracenic vibronic structure, while the emission spectrum is characterized by a broad, structureless band. Similar observations have been reported for 9-anthracenecarboxylic acid (9-ACA). ${ }^{41,26,27}$ The 
structureless emission of 9-ACA was ascribed to enhanced resonance interactions between the carboxylic acid group and the aromatic moiety, due to rotation of the functional group into the plane of the ring system following excitation. ${ }^{23}$

DFT calculations of the lowest energy configuration ground-state geometry of 9,10ADCA reveal a dihedral angle of $57^{\circ}$ between the carboxylic acid groups and anthracene plane. Comparably, 9-ACA was found to have a dihedral angle of $57^{\circ}$ and $88^{\circ}$ for the conjugate base, $9-\mathrm{ACA}^{-} \cdot{ }^{27}$ This ground-state configuration of 9-ACA is supported by crystallographic data. ${ }^{42}$ The non-planar geometry has been attributed to steric interactions between the acid group and the peri-hydrogens on the carbons in the 1 and 8 positions of the anthracene ring. ${ }^{23}$ The non-planar orientation prevents resonance interactions between the carboxylate groups and aromatic ring system, and gives rise to anthracene-like vibronic structure in the absorption spectrum. The inductive effects of the electron withdrawing, carboxylic acid groups result in the slight bathochromic shift observed in the absorption spectrum of 9,10-ADCA relative to anthracene. Deprotonation of the carboxylic acids reduces the strength of the inductive effects and consequently, the bathochromic shift in the absorption spectrum of $9,10-\mathrm{ADCA}^{2-}$ is smaller.

TDDFT calculations show that the dihedral angle, $\theta$, decreases to $\sim 28^{\circ}$ in the excited state of 9,10-ADCA (Figure 4f). Interestingly, as the carboxyl groups twist toward a more coplanar orientation, the anthracene ring also puckers. Previously, a $\theta$ of $\sim 33^{\circ}$ was calculated by TDDFT for 9-ACA in the excited state, but no ring distortion was reported. ${ }^{27}$ The distortion of the anthracene plane observed in 9,10-ADCA but not 9-ACA may be due to greater steric 
strain on the molecule imposed by the additional carboxylic acid. Puckering of the anthracene ring system upon rotation of the carboxyl groups disrupts the transition dipole moment across the short-axis. The large reconfiguration energies associated with this rotation and nuclear reorganization lower the energy of the excited-state, as evidenced by the large Stokes shift observed in the spectra of 9,10-ADCA. The more coplanar orientation allows for overlapping of the p-orbital of the acid group with the $\pi$-aromatic system of anthracene, introducing resonance interactions through delocalization of electron density from anthracene into the carboxyl substituents. This significantly perturbs the ${ }^{1} \mathrm{~A} \rightarrow{ }^{1} \mathrm{~L}_{a}$ transition dipole moment oriented along the short axis of anthracene, giving rise to a broad, structureless emission band. With increased resonance interactions, solvent-solute interactions have a greater contribution to the Stokes shift depending on the rate of rotation of the carboxyl groups relative to the rate of solvent reorganization as intramolecular nuclear reorganization occurs in the excited state. The mirror-image relationship between the absorption and emission spectra of the fully deprotonated 9,10- $\mathrm{ACA}^{2-}$ measured in basic solution indicates that the geometries of the ground and excited states of 9,10-ACA ${ }^{2-}$ are almost identical. Thus, the carboxylate groups remain decoupled from the aromatic system, resulting in vibronically structured emission. This is also supported by DFT/TDDFT calculations, which show little changes between the dihedral angles of $9,10-\mathrm{ADCA}^{2-}$ in the ground $\left(\theta=50^{\circ}\right.$, Figure S5c) and excited $\left(\theta=60^{\circ}\right.$, Figure $\left.\mathrm{S} 5 \mathrm{~d}\right)$ states.

\section{CONCLUSIONS}


Functionalization of anthracene with carboxylic acid groups results in perturbation of the photophysical properties depending on both the location of the groups and extent of their interaction with the aromatic ring system. In general, functionalization that extends the molecule along the molecular axes containing optical transitions of the same polarization affect the properties of that transition more significantly. For example, derivatization at the 2 and 6 positions extends the molecule along the longitudinal axis and enhances the longitudinally polarized ${ }^{1} \mathrm{~A} \rightarrow{ }^{1} \mathrm{~L}_{\mathrm{b}}$ transition, giving rise to intense ${ }^{1} \mathrm{~A} \rightarrow{ }^{1} \mathrm{~L}_{\mathrm{b}}$ absorption bands. In the 2,6-isomer, electron density remains evenly distributed in the molecule and vibronically structured absorption bands are observed. On the other hand, functionalization at the 1 and 4 positions, which lengthens the molecule along the short axis, gives rise to an uneven distribution of electron density along the polyaromatic ring system and results in broad, diffuse bands in both the absorption and emission spectra. The extent of interaction between the carboxylic acids and the anthracene ring is dictated by how the functional groups are orientated with respect to the anthracene plane. In 1,4-ADCA, the carboxyl groups lie coplanar with the parent anthracene, but in 9,10-ADCA, steric hindrances prevent coplanarity. As a result, there is greater overlap between the p orbitals of the acid groups in the 1 and 4 positions with the $\pi$ orbitals of anthracene, introducing a greater amount of charge transfer character in 1,4-ADCA compared to 9,10-ADCA. Accordingly, the photophysical properties of 1,4-ADCA are also sensitive to solvent-solute interactions and exhibit a dependence upon solvent polarity. The solvent $\mathrm{pH}$ also has a significant influence on the extent to which the functional groups interact with the ring system. In very basic solutions, where the carboxyl groups are completely deprotonated and cannot participate in 
resonance interactions, vibronically structured bands are observed in the absorbance spectra of all of the ADCA derivatives.

This work provides insight into the structure-property relationship of dicarboxylic anthracene derivatives and their fluorescent properties. An understanding of the effects of substitutions and local environment on the excited-state properties of anthracene can be used to further tune derivatives to obtain desired functionality. Such knowledge will aid in the design of next-generation optoelectronic materials.

\section{ACKNOWLEDGEMENTS}

This material is based upon work supported by the U.S. department of Energy, Office of Basic Energy Sciences under Award Number DE-SC0012446. The authors acknowledge Advanced Research Computing at Virginia Tech for providing computational resources and technical support that have contributed to the results reported within this paper.

\section{REFERENCES}

1.Zhu, M.Y., C. Yang (2013) Blue fluorescent emitters: Design tactics and applications in organic light-emitting diodes, Chem. Soc. Rev. 42, 4963-4976. 
2.Kaur, N.S., M. Singh, D. Pathakc, T. Wagner, J.M. Nunzi (2014) Organic materials for photovoltaic applications: Review and mechanism, Synth. Met. 190, 20-26.

3.Wakayama, Y., R. Hayakawa, H.S. Seo (2014) Recent progress in photoactive field-effect transistors, Sci. Technol. Adv. Mater. 15, 1019.

4.van de Linde, S.A., C. Franke, T. Holm, T. Klein, A. Löschberger, S. Proppert S. Wolter, M. Sauer (2013) Investigating cellular structures at the nanoscale with organic fluorophores, Chem. Biol. 20, 8-18.

5.Sidman, J.W. (1956) Electronic and vibrational states of anthracene, J. Chem. Phys. 25, 115121.

6.Shah, B.K.N., D.C. Neckers, J. Shi, E.W. Forsythe, D. Morton (2005) Photophysical properties of anthanthrene-based tunable blue emitters, J. Phys. Chem. A. 109, 7677-7681. 7.Moorthy, J.N., P. Venkatakrishnan, P. Natarajan, D.F. Huang, T.J. Chow (2008) De novo design for functional amorphous materials: Synthesis and thermal and light-emitting properties of twisted anthracene-functionalized bimesitylenes, J. Am. Chem. Soc. 130, 17320. 8.Tao, S.X., X. Zhang (2006) Efficient blue organic light-emitting devices based on novel anthracene derivatives with pronounced thermal stability and excellent film-forming property, Chem. Phys. Lett. 429, 622-627.

9.Swager, T.M., C.J. Gil, M.S. Wrighton (1995) Fluorescence studies of poly(pphenyleneethynylene)s: The effect of anthracene substitution, The J. Phys. Chem. 99, 48864893.

10.Platt, J.R. (1954) The box model and electron densities in conjugated systems, J. Chem. Phys. 22, 1448-1455. 
11.Klevens, H.B., J.R. Platt (1949) Spectral resemblances of cata-condensed hydrocarbons, J. Chem. Phys. 17, 470-483.

12.Platt, J.R. (1949) Classification of spectra of cata-condensed hydrocarbons, J. Chem. Phys. $17,484-495$.

13.Lackowicz, J.R. (2010) Principles of fluorescence spectroscopy 3rd Ed.; Springer Science+Business Media, LLC, pp. 32 .

14.Tigoianu, I.R., A. Airinei, D.O. Dorohoi (2010) Solvent influence on the electronic fluorescence spectra of anthracene, Rev. Chim. (Bucharest, Rom.) 61, 491-494. 15.Valeur, B., M.N. Berberan-Santos, (2012) Molecular fluorescence: Principles and applications; Wiley-VCH, pp. 77.

16.Berlman, I.B. (1971) Handbook of fluorescence spectra of aromatic molecules; 2d ed.; Academic Press: New York, pp. 57-59.

17.Melhuish, W.H. (1961) Quantum efficiencies of fluorescence of organic substances: Effect of solvent and concentration of the fluorescent solute, J. Phys. Chem. 65, 229-235.

18.Ware, W.R., B.A. Baldwin (1964) Absorption intensity and fluorescence lifetimes of molecules, J. Chem. Phys. 40, 1703.

19.Dawson, W.R., M.W. Windsor (1968) Fluorescence yields of aromatic compounds, J. Phys. Chem. 72, 3251 .

20.Lampert, R.A., L.A. Chewter, D. Phillips, D.V. O'Connor, A.J. Roberts, S.R. Meech (1983) Standards for nanosecond fluorescence decay time measurements, Anal. Chem. 55, 68-73. 21.Momiji, I., C. Yoza, K. Matsui (2000) Fluorescence spectra of 9-anthracenecarboxylic acid in heterogeneous environments, J. Phys. Chem. B. 104, 1552-1555. 
22.Abdel-Mottaleb, M.S.A., H.R. Galal, A.F.M. Dessouky, M. El-Naggar, D. Mekkawi, S.S. Ali, G.M. Attya (2000) Fluorescence and photostability studies of anthracene-9-carboxylic acid in different media, Int. J. Photoenergy. 2, 48-53.

23.Werner, T.C., D.M. Hercules (1969) Fluorescence of 9-anthroic acid and its esters.

Environmental effects on excited-state behavior, J. Phys. Chem. 73, 2005-2011.

24.Suzuki, S., T. Fujii, N. Yoshiike, S. Komatsu, T. Iida (1978) Absorption and fluorescence spectra of anthracenecarboxylic acids. I. 9-Anthroic acid and formation of excimer, Bull. Chem. Soc. Jpn. 51, 2460-2466.

25.Bazilevskaya, N., A. Cherkasov (1965) Two fluorescence bands of mesoanthracenecarboxylic acids and excimers, Appl. Spectrosc. 3, 412-416.

26.Ghoneim, N., D. Scherrer, P. Suppan (1993) Dual luminescence, structure and eximers of 9anthracene carboxylic acid. J. Lumin. 55, 271-275.

27.Rodriguez-Cordoba, W., R. Noria-Moreno, P. Navarro, J. Peon (2014) Ultrafast

fluorescence study of the effect of carboxylic and carboxylate substituents on the excited state properties of anthracene, J. Lumin. 145, 697-707.

28.Cabellero, A.G., A. K. Croft; S.M. Nalli (2008) Remote aromatic stabilization in radical reactions, Tetrahedron Lett. 49, 3613-3615.

29.Fontenot, S.A., V.M. Cangelosi, M.A.W. Pitt, A.C. Sather, L.N. Zakharov, O.B. Berryman and D.W. Johnson (2011) Design, synthesis and characterization of self-assembled $\mathrm{As}_{2} \mathrm{~L}_{3}$ and $\mathrm{Sb}_{2} \mathrm{~L}_{3}$ cryptands, Dalton Trans. 40, 12125-12131.

30.Garay, R.O., H. Naarmann, K. Mullen (1994) Synthesis and characterization of poly(1,4anthrylenevinylene). Macromolecules. 27, 1922-1927. 
31.Jones, N., Dimethyl 9,10-anthracenedicarboxylate: A aentrosymmetric transoid molecule, 67 (1945) 1922-1927.

32.Arient, J.P. (1973) Nitration and oxidation of anthraquinone dimethyl derivatives, Collect.

Czech. Chem. Commun. 39, 3117.

33.O’Connor, D.V., D. Phillips (1984) Time-correlated single photon counting; Academic Press: London, pp. 36-54.

34.Strickler, S.J., R.A. Berg (1962) Relationship between absorption intensity and fluorescence lifetime of molecules, J. Chem Phys. 37, 814-882.

35.Werner, T.C., D.M. Hercules (1970) Charge-transfer effects on the absorption and fluorescence spectra of anthroic acids, J. Phys. Chem. 74, 1030.

36.Imai, Y., K. Murata, N. Asano, Y. Nakano, K. Kawaguchi, T. Harada, T. Sato, M. Fujiki, R. Kuroda, Y. Matsubara (2008) Selective formation and optical property of a 21-Helical columnar fluorophore composed of achiral 2-anthracenecarboxylic acid and benzylamine, Cryst. Growth Des. 8, 3376-3379.

37.DiCesare, N., J.R. Lakowicz (2001) Spectral properties of fluorophores combining the boronic acid group with electron donor or withdrawing groups. Implication in the development of fluorescence probes for saccharides. J. Phys. Chem. A. 105, 6834-6840.

38.Becker, R.S. Theory and interpretation of fluorescence and phosphorescence; Wiley Interscience: New York, 1969; pp. 175-177.

39.Fitzgerald, L.J., R.E. Gerkin (1996)Anthracene-1,8-dicarboxylic acid, Acta Crystallogr. C. 52,1838 .

40.Fitzgerald, L.J., R.E. Gerkin (1997) Anthracene-1-carboxylic acid, Acta Crystallogr. C. 53, 1080. 